\title{
ARTICLE
}

\section{Value-based healthcare in mental health services}

\author{
Martin Roger Baggaley (i)
}

\begin{abstract}
Martin Roger Baggaley, FRCPsych, is a consultant general adult psychiatrist and former Executive Medical Director of South London and Maudsley NHS Foundation Trust (SLaM). After serving as a military psychiatrist in the British Army from 1985 until 1997 he was appointed to the post of consultant psychiatrist for the Lewisham and Guy's Mental Health Trust and senior lecturer in psychiatry for the Guy's, King's College and St Thomas's Hospitals' Medical and Dental School, London. $\mathrm{He}$ is interested in quality improvement, patient flow and the importance of clinical engagement in medical management.

Correspondence Dr Martin Roger Baggaley. Email: m.baggaley@btinternet.com
\end{abstract}

First received 24 Jun 2018 Final revision 6 Dec 2019 Accepted 18 Dec 2019

\section{Copyright and usage} (C) The Author 2020

\begin{abstract}
SUMMARY
In value-based healthcare (VBHC) value is defined as outcomes that matter to patients divided by the cost of achieving these outcomes. Value is measured for discrete medical conditions across the whole cycle of care. Data on the value achieved by different providers is openly shared. Providers increase value using quality improvement (0I) techniques to improve outcomes, reduce costs or both. Patients or commissioners choose the provider achieving the greatest value. Units should compete regionally or nationally. There are challenges to implementing such ideas in the mental health services in the UK. However, measuring outcomes, understanding costs and using $\mathrm{Ol}$ to drive up value may be possible without adopting the complete model that has developed in the context of a North American and acute hospital healthcare system.
\end{abstract}

\section{LEARNING OBJECTIVES}

After reading this article you will be able to:

- Define VBHC

- Explain the relevance to UK mental health services

- Understand the difference between $\mathrm{Ol}$ and VBHC

\section{DECLARATION OF INTEREST}

None.

\section{KEYWORDS}

Value-based healthcare; mental health outcomes; service delivery; quality improvement.

Value-based healthcare (VBHC) is a theory based on the work of Professor Michael Porter and Professor Elizabeth Teisberg from Harvard Business School (Porter 2006). At its core, VBHC is a way of driving quality improvement. Unlike existing quality improvement projects, which tend to be stand-alone initiatives, VBHC aims to improve care across whole services or organisations.

The key principle can be summed up in just one equation:

$$
\text { Value }=\frac{\text { Health outcomes }}{\text { Cost of delivering the outcomes }}
$$

The value (to patients) of a healthcare intervention is measured by dividing the outcome (that matters to the patient) by the cost of delivering that outcome. An example might be the treatment of a patient with depression. The desired outcome might be to return to being happy and to go back to work. This may require an assessment, 12 sessions of cognitive-behavioural therapy (CBT) and 6 months of an antidepressant. The CBT might cost $£ 1200$ and the monitoring of the medication $£ 1800$, making a total of $£ 3000$. The outcome might be defined as remission of symptoms, perhaps a score of less than 7 on the PHQ-9, a resumption of a relationship and a return to work.

Unfortunately, 'value' can be confused with 'values', i.e. moral or ethical values. There are also clarifications required over what is meant by a health outcome and how to measure the cost. There is a requirement to measure outcomes that matter to patients, instead of outcomes that matter to clinicians: patients may have little intrinsic interest in their MADRS score. There is a further refinement to understand what the costs are over the whole cycle of a patient's care. Interestingly, Porter $\&$ Teisberg do not consider patient satisfaction as a health outcome for this purpose (Porter 2006).

The concept of VBHC tends to be closely linked with quality improvement. To improve value one needs to get either better outcomes for the same cost or similar outcomes for less cost. One way of improving value is to stop doing things that do not improve outcomes that matter for patients. There is a considerable amount of waste in healthcare. It is estimated that $30 \%$ of healthcare interventions in Australia are wasteful (Organisation for Economic Co-operation and Development 2017). Waste can be caused by making the wrong diagnosis, giving the wrong treatment or both.

Most case examples given by Porter $\&$ Teisberg (2006) are from acute hospital care, and VBHC has been most frequently implemented in acute hospital settings. There are relatively few examples of the use of VBHC techniques in mental healthcare. However, there is as much need to improve 'value' in mental as in physical healthcare.

VBHC suggests a more integrated and holistic approach to healthcare. This includes the integration of mental with physical healthcare. This may be where some of the greatest increase in value may be found. 
VBHC emphasises the importance of embracing and rewarding innovation. Mental healthcare may not be very equipment intensive but nevertheless innovation could be used to a far greater extent. For example, logistics management software used to manage retailers' home deliveries or minicab operations might be used to more effectively manage community nurses undertaking home visits; or there might be greater use of SMS texts, telephone calls and emails for patient contact rather than face-to-face contact (Williams 2017).

\section{Competition as driver of improved value}

\section{Value-based competition}

Porter \& Teisberg (2006) explicitly assume that competition is one of the principal drivers to improve value. Although theoretically there is an element of competition in mental health service delivery in the UK, it is very limited. They suggest that there is a difference between value-based and zero-sum competition. In value-based competition, outcomes improve for patients, costs reduce and good providers expand and grow. In contrast, most healthcare systems in the UK are operated on the basis of zero-sum competition, in which the same pie is redistributed between providers. This is how most competition in the National Health Service (NHS) operates. Providers compete for the same business, often at reduced cost. This divides value rather than increases it (Rahman 2015).

In zero-sum competition, increased value is hoped to be achieved by either shifting costs or reducing costs by restricting services. So, in the NHS the clinical commissioning groups (CCGs) and NHS England try to shift costs onto providers rather than truly improve value. Providers and CCGs try to reduce costs by rationing or restricting access to services (Robertson 2016).

\section{Economies of scale and bargaining power}

Other strategies to increase value include increasing the size of the provider (i.e. hospital mergers or taking over larger geographical areas) to make efficiency because of size or to try to drive down price by using the size of the organisation as a bargaining tool.

\section{Service delivery: integrated practice units}

Porter \& Teisberg (2006) suggest that competition should be for a particular medical condition across 'the full cycle of care'. They advocate organising delivery along service lines to treat, say, diabetes with all relevant clinicians brought together in a functional unit. Such units would specialise in a particular disorder or part of the pathway and become expert at delivering this type of care. Such care should be patient centred and integrated. They call these units condition-specific integrated practice units (IPUs).

It is not as easy to introduce condition-specific IPUs in mental healthcare, where individual diagnoses do not always correlate well with functional impairment or treatment need. This issue has been a cause of difficulty in designing reimbursement systems for mental healthcare (de Figueiredo 1985). For example, a patient with bipolar affective disorder experiencing a manic episode may need a similar treatment approach to a patient with schizophrenia. One could then have an IPU for 'psychosis'. However, there may be patients with bipolar affective disorder who have persistent depression who would be best treated by an IPU for 'depression'. Currently, most mental healthcare services are organised by age (i.e. child and adolescent, working age, and old age). One could envisage an IPU for eating disorder or borderline personality disorder offering services across the lifespan. An early onset team is a form of IPU although perhaps not strictly condition specific.

\section{Outcome data as a driver of value-based competition}

Porter \& Teisberg (2006) emphasise the central importance of information to drive competition. This information must include transparent data on the outcomes of treatment (outcomes that matter to patients). They point out that clinicians cannot compare their performance against others without the right outcome data. Furthermore, patients cannot choose the best provider without knowing the results achieved by different units. The evidence across acute care shows that publishing and disseminating outcomes from competing providers drives up quality (Shekelle 2008).

Value-based competition means focusing not just on lowering costs but also on providing value for patients. The actual competition should be on results and on medical conditions over the full cycle of care. Porter $\&$ Teisberg argue that, in the long run, high-quality care would reduce costs. In my view, many NHS clinicians do not truly accept this point and consider it some form of management 'double-speak'. This may be because NHS clinicians are intrinsically cynical and dismissive of management initiative. They also may have experienced real cuts to services disguised as 'improvement'. However, there is considerable evidence to support the contention that there is only a weak association between cost and quality (Hussey 2013). If a patient is diagnosed accurately and treated with the most effective evidence-based intervention, 
improves as quickly as possible and is then kept well away from costly in-patient beds, it is far cheaper than a poorer quality pathway (Newman-Toker 2013).

Porter $\&$ Teisberg suggests that 'value' must be driven by provider experience, scale and learning at the level of the medical condition concerned. This is very much an argument for specialisation and hard to achieve in typical NHS mental health trusts when most mental health professionals are generalists. Indeed, in the past few years, in response to reducing resources community mental health teams have become less not more specialised.

Competition should be regional and national and not just local. Results information to support value-based competition must be widely available. Innovations that increase value must be strongly rewarded. There may not be many dramatic innovations in treating acute psychosis. However, simply ensuring that the most appropriate protocols are used, for example the early use of clozapine, may result in significant cost savings (Lawrie 1998). Cutting costs may lead to long-term expenditure. For example, if assessments are largely performed by less experienced and less qualified mental health professionals, there may be errors in diagnosis and delays in instigating effective treatment.

In the NHS in general, and perhaps mental health in particular, we are a long way from achieving this. The majority of initiatives focus on cost savings. There is little real competition among mental health providers and most competition, if it exists, is local.

\section{A practical example: first-episode psychosis across the care cycle}

An example of treating a medical condition across the whole cycle of care might be a first episode of psychosis. The outcome measure most commonly used by the NHS in first-episode psychosis is the Health of the Nation Outcome Scales (HoNOS) but collection of data-sets, especially paired outcome measures, is patchy (Macdonald 2015). There is a clear requirement to agree a more comprehensive and useful set of outcome measures for the illness. Then there is the need for such measures to be collected and disseminated. The Royal College of Psychiatrists has a working group establishing a common set of outcome measures for this purpose and the International Consortium for Health Outcomes Measurement (ICHOM) has also established outcome measure sets for particular mental health conditions.

It is often hard for clinicians to accept that high-quality care is cheaper. However, if excellent diagnostic services quickly diagnose first-episode psychosis and begin treatment to achieve early remission, large sums of money may be saved by avoiding subsequent hospital admissions. A major problem is the absence of national and regional competition. This is due to the fact that much mental healthcare is linked with local authority social care. Also, there are relatively few elective admissions and a greater proportion of emergency admissions, many presenting via the emergency department or a place of safety (Crisp 2017). It is much easier to send a patient from London to Newcastle for a hip replacement if the unit in the north-east produces much better outcome results than it is to send a patient with first-episode psychosis.

Results need to be 'real' outcomes that are important for patients. In the NHS excellence is usually judged not on results but on compliance with particular nationally imposed external pathways and protocols.

\section{Defining conditions and care cycles}

The question of what constitutes a 'medical condition' in terms of mental health services is difficult (Jablensky 2016). It can, however, be defined, especially for specialised services. So, autism or eating disorder could be good examples of services that could be compared on results across the whole cycle of care. Treatment-resistant severe affective disorder also could be looked at in this way. Some trusts have tried to organise services along disease lines; for example, the South London and Maudsley NHS Foundation Trust created clinical academic groups for psychosis, mood anxiety, personality disorders and so on. This can work well in some situations but it can complicate service delivery in particular local services that work better when integrated.

It can be difficult to decide when a care cycle begins or ends. Many of the patients in secondary mental healthcare have long term conditions and are cared for in the service for many years. In such cases a care cycle might be defined by a particular time duration, for example a year of care.

Value is said to be improved by treating one thing well rather than treating everything. Many mental health clinicians in the UK treat everything that comes their way. There would be advantages in having clinicians who just treat one particular condition. This is possible in some national and tertiary clinics but the problem is again the difficulties of treating people on a regional or national basis.

\section{Requirements for delivering VBHC in UK mental health services}

A criticism of Porter \& Teisberg's theories on VBHC (Box 1) would be that they are both USA and acutemedicine centric. It is therefore not certain that they 
BOX 1 Key requirements for value-based healthcare (VBHC)

- Measuring outcomes that matter for patients

- Measuring the costs of achieving these outcomes

- Focus on a particular medical condition

- Focus on the whole cycle of care

can be easily applied to the NHS in the UK in general and to mental healthcare in particular. However, There have been some encouraging initiatives, for example from St Andrew's Healthcare, a charity providing specialist mental healthcare for young people and adults (Wallang 2018). There is considerable evidence that organisations that pursue systematic quality improvement and the concepts of value-based medicine provide better and safer care. Both the East London (Shah 2018) and the South London and Maudsley NHS Foundation Trusts have embarked on such an approach (both in partnership with the Institute of Healthcare Improvement) and have demonstrated early benefits. The experience of organisations in the USA such as the Cleveland Clinic and Intermountain Healthcare (Porter 2006) shows that this is a longterm endeavour and the real benefits are achieved several years into the programme.

There are several obvious challenges to implementation in the NHS, the first of which is that routine outcome measures (whether they equate to outcomes that matter to patients or not) are not in use across many mental health settings in the UK. Second, few pathways or interventions have been properly costed. Third, there is a lack of patient choice and practical difficulties in receiving care from another provider in a different locality.

\section{Determining outcomes that matter to patients}

A typical method is to hold workshops with patients, carers and clinicians to explore what are the outcomes that matter to patients and determine ways of measuring them. It is important to use outcome measures that are agreed by the majority of providers and that are robust and validated. It is also important to use measures that are easy to collect and score. Such outcome measures must be transparent and fed back to - and owned by - the clinical teams, who must take responsibility for understanding how they compare to other teams and strive to improve their outcomes. There are a number of organisations and teams working to support the development of standard frameworks for outcomes, such as ICHOM (mentioned above), the ConsensusBased Standards for the Selection of Health
Measurements Instruments (COSMIN) team and the Core Outcome Measures in Effectiveness Trials (COMET) initiative.

Outcome measurement is generally underdeveloped in mental health services. There are some exceptions; for example, the Improving Access to Psychological Therapies (IAPT) programme has a very thorough and systematic system (Clark 2018). Many trusts have made progress in collecting HoNOS data. However, it is unclear whether such measures capture outcomes that matter to patients. Even if they do, there are not adequate systems to compare outcomes both within a trust and then across trusts. There are often concerns raised that there would be incentives to 'game' outcomes. In practice, there is little evidence that this is widespread. It would be relatively easy to introduce audits and external reviews to police the system. There have been some useful developments in collecting quality of life measures in NHS trusts, for example the use of DIALOG (a computer-mediated procedure structuring routine patient-clinician communication) at East London NHS Foundation Trust (Priebe 2007) or Recovering Quality of Life (ReQOL, a generic self-reported outcome measure) at Berkshire NHS Foundation Trust (Keetharuth 2018).

\section{Measuring the cost of interventions and payment systems}

The understanding and measuring of the costs of interventions at an individual patient level are just as problematic as the measurement of outcomes.

In some teams, it might be possible to roughly estimate by dividing the cost of running the team by the case-load. However, to really move to VBHC it is necessary to be able to measure cost at a patient level. Of course, there needs to be clear description of what the intervention is. Some psychological interventions are manualised but many are not. What actually goes on in a follow-up session with a consultant psychiatrist (Killaspy 2006)? What sort of therapy is happening in a psychology session? What is the care coordinator actually doing? There is a need for common description of interventions, their cost and the grade of staff required to deliver them. Kaplan \& Anderson developed a methodology to define the cost of individual elements in an intervention (Kaplan 2004). A costeffectiveness analysis in a clinic in Stockholm, Sweden, combined time-driven activity-based costing (TDABC) with clinical outcome to measure value in the $\mathrm{CBT}$ treatment of depression (El Alaoui 2016).

The type of payment system is crucial. The intention of moving to an outcome-based commissioning 
approach would support and be consistent with a value-based healthcare approach. This would involve issuing long-term contracts with payment for outcomes rather than activity. There would still be a requirement to measure outcomes that matter to patients. There might not necessarily be direct competition with other organisations but the performance of competitor organisations would influence the reimbursement of the providers.

There would need to be a set of nationally agreed outcome measures and standard units of cost. The commissioning arrangements would need to align to these outcome measures such that providers were rewarded for achieving good outcomes. The providers would need to capture the outcome measures achieved and there would need to be systems to make the results (and costs to achieve them) open and transparent internally to the organisation and externally to other providers in potential competition, as well as to commissioners and patients.

\section{Facilitating patient choice and movement to better providers}

For VBHC as Porter \& Teisberg envisage to truly work in the NHS, patients (and/or commissioners) would need to be able compare value (outcomes that matter to patients/cost to achieve those outcomes) for a specific condition across the whole cycle of care. Having compared value, they would need to be able to access the services that offered the best value.

This is clearly not practicable in many cases owing to geographical constraints. So, if a crisis resolution/ home treatment team in Newcastle were to offer outstanding value, it would not be possible for patients in London to access that team. However, it might work for some services in some areas.

Specialised regional or national services could operate on VBHC principles. For example, mother and baby, forensic, low secure and intensive care in-patient facilities that demonstrated outstanding value could develop and attract patients.

It is conceivable that modern technology such as telemedicine could allow organisations to operate over wider geographical areas and allow some type of franchise model such that patients could choose services, say, from Northumbria operated by an affiliate in London (Williams 2017).

\section{Locally provided services and specialised mental health trusts}

Should trusts specialise in particular things they are good at? Local services are expected to provide care for all and this is likely to remain so for general community mental health teams. However, in specialised care it could be possible for some trusts to offer, for example, eating disorder, forensic or perinatal services regionally or nationally. There is an issue, however, about interfaces and the interoperability of information systems to contend with this specialisation. The experience of providing some services separate from local community services (e. g. tier 4 child and adolescent mental health services) has not been positive.

The opposite problem is that the services offered may be too narrow and not be sufficiently integrated with, for example, primary or social care. So, a very specific service line might only treat, say, an eating disorder but not comorbid mood disorders. There are examples of VBHC in mental health services being based on an integrated primary care model.

\section{The role of quality improvements}

Determining outcomes (that matter to patients) and the costs of achieving such outcomes is only part of the challenge. There is some evidence that simply measuring outcome and cost can drive up value (Campanella 2016). However, major transformation requires that systems and methods improve value, either by improving outcomes, reducing cost or both. There may be a number of steps in the patient pathway that do not add value, i.e. improve outcomes that matter to patients. These need to be identified and stopped. Then the time saved needs to be used either to do things that do add value, i.e. improve outcomes in individual patients, or to see more patients (thus increasing productivity).

There are a number of well-researched quality improvement (QI) methods, such as lean, the Toyota Production System, the Model for Improvement and the 'plan-do-study-act' (PDSA) cycle (Healthcare Quality Improvement Partnership 2015). It probably does not matter which is selected provided that the clinical teams have the necessary knowledge and resources to engage in QI.

The challenge is that becoming a VBHC organisation requires a massive cultural change throughout, from the frontline staff to the board of directors. This requires persistence and determination of leadership, resources and many years or effort. The history of many such initiatives in the NHS is an attempted top-down implementation by an enthusiastic few which is abandoned in a few years to be replaced by the next big idea.

\section{Summary}

\section{What is VBHC?}

Value-based healthcare (VBHC) is an ideology that offers a potential solution to the universal healthcare conundrum of an inexorable increase in demand due to demographic change in a world of constrained 
resources and ever-increasing expectations. At its heart is a simple relationship of defining value as outcomes that matter to patients divided by the cost of achieving these outcomes. Having defined value, providers then resolutely and persistently pursue increasing value by improving outcomes and reducing the costs of producing such outcomes. This can be achieved by using protocols, cutting out wasteful practice, providing evidence-based interventions and exploiting innovation. The outcomes and costs must be condition specific and measured across the whole cycle of care. These outcomes and costs must be openly published and shared with other providers and patients. The system should allow super-specialisation and make care available regionally and nationally. Patients and commissioners should be allowed to choose those providers who demonstrate excellent value. In a free market economy, such providers would expand and grow while mediocre providers would shrink and eventually close.

\section{Is it transferable to UK mental healthcare?}

There are objections that VBHC is a USA- and acutecentric approach incompatible with the culture and ethos of the NHS in the UK (Box 2). There are clearly major challenges in measuring and collecting outcomes that matter to patients and in measuring patient-level costs. There is a lack of well-embedded QI methods and processes. Most UK mental healthcare is provided by local services that have an effective monopoly and do not treat individual medical conditions across the cycle of care.

There are some NHS services in which it would be relatively easy to adopt the Porter $\&$ Teisberg model in its entirety and it would be beneficial to do so. Most obviously, relatively stand-alone specialised services such as eating disorders could become condition-specific IPUs. It would require consensus on outcomes (that matter to patients) and agreement on packages of intervention, which would be costed to allow different IPUs to compare their achievement in terms of value. It would require the ability of successful IPUs to increase their capacity and a willingness for commissioners to respond to improved values.

\section{BOX 2 Challenges in implanting value-based healthcare (VBHC) in UK mental health services}

- Underdeveloped outcome measurement

- Poor understanding of cost

- Care is not organised by particular condition

- Care is not organised across the whole cycle of care

- Most services cannot compete regionally or nationally
There are other units/teams that could be considered condition specific, such as challenging behaviour, mother and baby, and early-onset psychosis. These might be compared on value, even if geography makes it is difficult to move patients to teams that are producing increased value.

\section{Would it benefit the NHS?}

Porter \& Teisberg (2006) argue that it is irrefutable that measuring and publishing healthcare outcomes improves quality. The added benefit of a VBHC approach is that it links cost to outcome and emphasises the importance of competition. It is indeed likely that, if NHS mental health providers could manage to measure and compare outcomes that matter to patients, measure and compare costs, and implement a robust and systematic QI programme, the standard of care would improve considerably and costs would reduce. VBHC as envisaged by Porter $\&$ Teisberg might need adaptation to suit the NHS and mental healthcare, but even in a modified form it has much to commend it.

\section{References}

Campanella P, Vukovic V, Parente P, et al (2016) The impact of Public Reporting on clinical outcomes: a systematic review and meta-analysis. BMC Health Services Research, 16: 296.

Clark DM (2018) Realizing the mass public benefit of evidence-based psychological therapies: the IAPT program. Annual Review of Clinical Psychology, 14: 159-83.

Crisp N, Smith G, Nicholson K (2017) Old Problems, New Solutions: Improving Acute Psychiatric Care for Adults in England. Commission on Acute Adult Psychiatric Care.

de Figueiredo JM, Boerstler $H$ (1985) DRGs and reimbursement for inpatient psychiatry. Comprehensive Psychiatry, 26: 567-72.

El Alaoui S, Lindefors N (2016) Combining time-driven activity-based costing with clinical outcome in cost-effectiveness analysis to measure value in treatment of depression. PloS One, 11(10): e0165389.

Healthcare Quality Improvement Partnership (2015) A Guide to Quality Improvement Methods. HOIP.

Hussey PS, Wertheimer S, Mehrotra A (2013) The association between health care quality and cost: a systematic review. Annals of Internal Medicine, 158(1): 27-34.

Jablensky A (2016) Psychiatric classifications: validity and utility. World Psychiatry, 15: 26-31.

Kaplan R, Anderson S (2004) Time-driven activity-based costing. Havard Business Review, November: 131-8.

Keetharuth AD, Brazier J, Connell J, et al (2018) Recovering Quality of Life (ReQoL): a new generic self-reported outcome measure for use with people experiencing mental health difficulties. British Journal of Psychiatry, 212: $42-9$

Killaspy H (2006) Psychiatric out-patient services: origins and future. Advances in Psychiatric Treatment, 12: 309-19.

Lawrie SM (1998) Risperidone and clozapine were more cost effective than haloperidol and chlorpromazine in patients with schizophrenia: commentary. Evidence-Based Mental Health, 1(2): 64.

Macdonald AJD, Fugard AJB (2015) Routine mental health outcome measurement in the UK. International Review of Psychiatry, 27: 306-19.

Newman-Toker DE, McDonald KM, Meltzer DO (2013) How much diagnostic safety can we afford, and how should we decide? A health economics perspective. BMJ Quality and Safety, 22(suppl. 2): 11-20.
MCO answers

$1 \mathrm{c} \quad 2$ a 3 b 4 a 5 b 
Organisation for Economic Co-operation and Development (2017) Tackling Wasteful Spending on Health. OECD Publishing.

Porter ME, Teisberg EO (2006) Redefining Health Care: Creating ValueBased Competition on Results. Harvard Business Review Press.

Priebe S, McCabe R, Bullenkamp J, et al (2007) Structured patientclinician communication and 1-year outcome in community mental healthcare: cluster randomised controlled trial. British Journal of Psychiatry, 191: 420-6.

Rahman F, Kiernan F (2015) Competition in the UK National Health Service. HealthManagement, 15(2).

Robertson, R (2016) NHS rationing under the radar. The King's Fund blog, 17 August (https://www.kingsfund.org.uk/blog/2016/08/nhs-rationingunder-radar)
Shah A, Course S (2018) Building the business case for quality improvement: a framework for evaluating return on investment. Future Healthcare Journal, 5: 132-7.

Shekelle PG, Lim Y-W, Mattke S, et al (2008) Does Public Release of Performance Results Improve Quality of Care? A Systematic Review. Quest for Quality and Improved Performance (QOUIP), The Health Foundation.

Wallang P, Kamath S, Parshall A, et al (2018) Implementation of outcomes-driven and value-based mental health care in the UK. British Journal of Hospital Medicine, 79: 322-7.

Williams OE, Elghenzai S, Subbe C, et al (2017) The use of telemedicine to enhance secondary care: some lessons from the front line. Future Healthcare Journal, 4: 109-14.

\section{MCOs}

Select the single best option for each question stem

\section{As regards $\mathrm{VBHC}$ :}

a it was first proposed by Michael Porter and Elizabeth Teisberg from Harvard Medical School

b patient satisfaction scores are an important component of outcomes that matter to patients

c value-based competition is a key principle in VBHC

$\mathrm{d}$ competition is best if local

e successful providers should not grow in size.
2 As regards value (to patients) of healthcare interventions:

a value is increased by stopping wasteful practices

b value is decreased by reducing costs

c value is increased by improved patient satisfac tion scores

d it has been estimated that up to $50 \%$ of medical interventions in Australia are wasteful

e value is decreased by innovation.

3 As regards $\mathrm{VBHC}$ :

a $\mathrm{VBHC}$ refers to ethical and moral values in healthcare

b VBHC initiatives have begun in several UK mental health trusts

c lean methodology is the only $\mathrm{Ol}$ methodology in VBHC

d VBHC is best implemented in small chunks of the clinical pathway

e value is reduced by increased outcomes
4 The following applies to VBHC:

a innovation is an important concept in VBHC

b IPU is a term for independent or private providers

c outcomes should be determined by clinicians

d outcomes need to be kept confidential for commercial reasons

e different providers can use any outcome measure they choose.

5 According to the principles of VBHC:

a providers must provide all possible clinical pathways

b providers should only provide pathways at which they excel

c providers should not be allowed to fail

$\mathrm{d}$ the only acceptable outcome measures are those of importance to researchers

e zero-sum competition is best. 